\title{
NO TRADE AND YES TRADE THEOREMS FOR HETEROGENEOUS PRIORS
}

\author{
ALIA GIZATULINA AND ZIV HELLMAN
}

\begin{abstract}
We show that even under heterogeneous priors the classical no trade theorem obtains. However, speculative trade becomes mutually acceptable if at least one party to the trade puts at least some slight probability on the other party being irrational. We also derive bounds on disagreements in the case of heterogeneous priors and $p$-common beliefs.
\end{abstract}

\section{INTRODUCTION}

One commonly hears it said that 'if there is a common prior among agents, then no trade is possible between them'; this is indeed true. It is, however, also not any less common to hear the (erroneous) inverse to that statement, that is, a claim that 'if priors are not common, then any unbounded volume of trade may ensue'. The goal of this paper is twofold: first we show that even if priors are not common (and there are arbitrarily large disagreements among agents about the expected value of a random variable), a corresponding version of the no-trade theorem obtains. Second, we extend to the case of heterogeneous priors a result due to Neeman (1996b) that shows the necessity of the existence of slight irrationality of each player for trade to occur. Specifically, we demonstrate that when agents do not share a common prior, for trade to occur it is sufficient that each agent $i$ believes that with some arbitrarily small probability other agents are irrational and play suboptimal strategies, while player $i$ ascribes probability one that himself he is perfectly lucid and plays the best-reply to others' suboptimal strategy.

The No Trade Theorem, as developed by Milgrom and Stokey (1982) and others (such as Rubinstein (1975); Tirole (1982); Dow et al (1990)), is considered one of the most surprising of the fundamental insights in the theoretical literature in recent decades. It states that under the assumption of common priors and ex-ante Pareto efficient allocations the arrival of private information will not induce further trade if the acceptability of a proposed

Date: This version, July 2, 2018. 
trade is common knowledge. Under its usual interpretation as a "no speculation' result, it has been especially perplexing because it stands in stark contrast to the immense volume of speculative trade observed daily in security markets.

The No Trade theorem is usually presented as building upon two other surprising results, the No Disagreement theorem and the No Betting theorem. These theorems essentially state that if agents have common priors, they will never take opposite sides of any commonly acceptable proposed bet even after receiving private information. Moreover, the no betting result is bi-directional, i.e., a common prior precludes betting while heterogeneous priors imply the existence of agreeable bets (i.e. that both agents would agree to disagree about the expected value of a random variable). It is this that leads to the common but erroneous argument that the No Trade theorem is also dependent on the common prior assumption. Indeed, the counter-intuitive quality of the No Trade theorem contrasted with observed trade volumes is sometimes adduced as an argument against assuming common priors in 'the real world'. In this view, dropping the common prior assumption puts the theory back in harmony with the empirical existence of speculative trade.

We show here that, in fact, the No Trade result is independent of the question of whether or not priors are common; it follows solely from the combination of ex-ante Pareto efficiency, common knowledge of rationality, and common knowledge of an agreed trade. The proof of this is, somewhat surprisingly, almost entirely a reprise of the proof of the original No Disagreements Theorem of Aumann (1976).

The impact is that attempting to explain the existence of large volumes of trade by supposing that traders do not start from common priors is misguided. The alternative that remains is to weaken the assumption common knowledge of rationality to restore the possibility of mutually agreed trading. ${ }^{1}$

The idea of attaining trade results by moving away from common knowledge or rationality is not entirely new. Neeman (1996b) presents a model with common priors in which speculative trade is possible between traders with only common $p$-belief of rationality. However, the model in that paper must assume that each trader ascribes some positive probability to himself being irrational at some state, making its interpretation difficult. It would seem much more natural to postulate that each trader accepts a trade based

\footnotetext{
${ }^{1}$ Other possible explanations that have appeared in the literature include: noisy traders, bounded rationality, and Knightian uncertainty.
} 
on a belief that others are irrationally acquiescing to the terms of the trade, while being certain that he is immaculately rational.

Neeman (1996b), noting the problematic aspect of all agents ascribing a non-zero probability of self-irrationality, conjectures that with heterogeneous priors trade can occur with each trader assigning positive probability only to the other trader being irrational, leaving himself perfectly rational. We show here that this is indeed true, and that furthermore it suffices for one trader to ascribe positive probability, as small as desired, of the other trader following an irrational strategy at only some state for trade to occur.

\section{PRELIMinARIES}

2.1. Partitions. Let $I$ be a finite set of players. Let $\Omega$ be a compact metric state space, endowed with a topology $\tau$. Denote by $\Sigma$ the Borel $\sigma$-field of $\Omega$ generated by topology $\tau$; the elements of $\Sigma$ will be referred to as events. Assume that the corresponding space of measures $\Delta(\Omega)$ is endowed with the topology of weak convergence.

For each $i$, the collection $\Pi^{i} \equiv\left\{\Pi^{i}(\omega) \mid \omega \in \Omega\right\}$ is a partition of $\Omega$, such that each partition element $\Pi^{i}(\omega)$ is measurable. We assume that this partition is finite for all $i . \Pi=\left(\Pi^{i}\right)_{i \in I}$ is called a partition profile of the set of players $i \in I$. Denote by $\mathcal{F}^{i}$ the $\sigma$-field generated by $\Pi^{i}$, so that $\mathcal{F}^{i}$ consists of all unions of elements of $\Pi^{i}$. It follows that $\mathcal{F}^{i} \subset \Sigma$.

The meet of $\Pi$, denoted $\wedge \Pi$, is the partition that is the finest among the partitions that are simultaneously coarser than all the partitions $\Pi^{i}$. We will denote by $\Pi(\omega)$ the element of $\wedge \Pi$ containing $\omega . \Pi$ is called connected when $\wedge \Pi=\{\Omega\}$.

2.2. Knowledge. We say that player $i$ knows event $E$ at state $\omega \in \Omega$ if $\Pi^{i}(\omega) \subseteq E$. Let $K_{i}(E)$ be the event that $i$ knows $E$, that is,

$$
K_{i}(E):=\left\{\omega \mid \Pi^{i}(\omega) \subseteq E\right\} .
$$

An event $C$ is common knowledge at $\omega$ if there exists an event $E$ such that $\omega \in E \subseteq C$ and $E \in \mathcal{F}^{i}$ for all $i$. There is an alternative characterisation of common knowledge. Call an event $E$ an self-evident if for each $i$,

$$
E \subseteq K_{i}(E)
$$

i.e., an event is self-evident if when it occurs everyone knows it, regardless of any private information of signalling. Then, by a theorem due to Monderer and Samet (1989), an event $C$ is common knowledge at $\omega$ if and only if there exists a self-evident event $E$ such that $\omega \in E$ and for all $i$,

$$
E \subseteq K_{i}(C)
$$


2.3. Types and Prior Distributions. Let a partition profile $\Pi$ be given. For each $i \in I$ and $\omega \in \Omega$, let $t_{i}(\omega)$ be a probability measure on $\Omega$, such that:

(a) $t_{i}(\omega)\left(\Pi_{i}(\omega)\right)=1$;

(b) for each $\omega^{\prime} \in \Pi^{i}(\omega), t_{i}\left(\omega^{\prime}\right)=t_{i}(\omega)$.

A function $t_{i}: \Omega \rightarrow \Delta(\Omega)$ thus defined is called a type function and $t_{i}(\omega)$ is a type of $i$ at $\omega \in \Omega$. Throughout the paper we assume that $t_{i}(\omega)$ is a continuous function and each $i \in I$ has a regular non-atomic Borel probability measure $t_{i}(\omega)$ on $\Omega$ at every state $\omega \in \Omega$.

A type space is then a tuple

$$
\mathcal{T}=\left\{I, \Omega,\left(\Pi_{i}, t_{i}\right)_{i \in I}\right\},
$$

where $t_{i}$ is a type function for each $i$. We will say that a type space $\mathcal{T}$ is connected if its underlying partition profile $\Pi=\left(\Pi^{i}\right)_{i \in I}$ is connected.

A probability measure $\mu^{i} \in \Delta(\Omega)$ is a prior for player $i$ if for every event $E \in \Sigma, \mu^{i}(E)=\int_{\Omega} t^{i}(\omega)(\cdot)(E) d \mu^{i}(\cdot)$. In other words, $\mu^{i}$ is a prior if $i$ 's types $t^{i}(\omega)$ are the posteriors of $\mu^{i}$ conditional on $i$ 's information function $t^{i}$. A probability measure $\mu \in \Delta(\Omega)$ is a common prior if it is a prior for each $i \in I$. We will not assume the existence of common priors here unless it is explicitly stated that one exists.

For a measurable random variable $f$ over $\Omega$, the prior expectation of a player $i$ with respect to a prior $\mu^{i}$ will be denoted $E_{\mu^{i}} f=\int_{\Omega} f(\cdot) d \mu^{i}(\cdot)$. The posterior expectation of $f$ by player $i$ at a state $\omega$ will be denoted

$$
E_{i}\left(f \mid \Pi^{i}(\omega)\right)=\int_{\Omega} f(\cdot) d t^{i}(\omega)(\cdot) .
$$

The expected value of an event $H$ will be understood to be the expected value of the standard characteristic function $1^{H}$ which is defined as:

$$
1^{H}(\omega)= \begin{cases}1 & \text { if } \omega \in H \\ 0 & \text { if } \omega \notin H\end{cases}
$$

Given the type space $\mathcal{T}$, denote the set of all priors of player $i$ by $W^{i}(\mathcal{T})$, or simply by $W^{i}$ when $\mathcal{T}$ is understood. ${ }^{2}$ In general, $W^{i}$ is a set of probability distributions, not a single element; it is, in fact, a closed and convex set (Samet (1998), Heifetz (2006)).

\footnotetext{
${ }^{2}$ Strictly speaking, the set of priors of a player $i$ depends solely on $i$ 's type function $t^{i}$, not on the full type profile $\mathcal{T}$. However, since we are studying connections between sets of priors of different players, we will find it more convenient to write $W^{i}(\mathcal{T})$.
} 
2.4. Beliefs. To articulate players' mutual beliefs and knowledge we make use of the following the generalization of the concept of common knowledge due to Monderer and Samet (1989). In particular, suppose $\mathcal{T}$ is a type space and that for each $i, \mu^{i}$ is a prior for player $i$. Then player $i$-believes event $E$ at $\omega$ if $\mu^{i}\left(E \mid \pi^{i}(\omega)\right) \geq p$. The event that ' $i p$-believes $E$ ', denoted $B_{i}^{p}(E)$ is

$$
B_{i}^{p}(E):=\left\{\omega \mid \mu^{i}\left(E \mid \Pi^{i}(\omega)\right) \geq p\right\} .
$$

An event $E$ is called (heterogenously) ${ }^{3}$ evident $p$-belief if for each $i \in I$

$$
E \subseteq B_{i}^{p}(E)
$$

In words, an event is an evident $p$-belief event if when it occurs everyone believes that it occurs with probability at least $p$, regardless of private information or signalling.

An event $E$ is (heterogenously) common $p$-belief at $\omega$ if there exists an evident $p$-belief event $F \ni \omega$ such that for all $i \in I$,

$$
F \subseteq B_{i}^{p}(E)
$$

It is straightforward to show that for every $0 \leq p \leq 1$, player $i$, and $E \in \Sigma$, one has $B_{i}^{p}(E) \in \mathcal{F}^{i}$, i.e. $B_{i}^{p}$ is measurable with respect to $\mathcal{F}^{i}$. containing $\omega$.

Finally, we note that when $p=1$, the above definition corresponds to 'knowledge' rather than 'belief'.

\section{Common Priors: No Betting And No Trade}

3.1. No Disagreements and No Agreeable Bets. The main characterisation of the existence of common priors in the literature is based on the concept of agreeable bets.

We suppose throughout this section that a connected type space $\mathcal{T}$ is given, along with a profile of priors $\left\{\mu_{i}\right\}_{i \in I}$.

Definition 1. An $n$-tuple of random variables $F:=\left\{f^{1}, \ldots, f^{n}\right\}$ over $\Omega$, one for each player $i \in I$, is a bet if $\sum_{i=1}^{n} f^{i}(\omega)=0$ for all $\omega \in \Omega$.

In our model, when offered to participate in a bet $F$ at a state $\omega$, a player may choose an action from a set of possible actions

$$
A=\{\text { 'accept', 'reject', 'refrain' }\} \text {. }
$$

\footnotetext{
${ }^{3}$ We use the term heterogenously here to refer to the possibility that the players may not share a common prior.
} 
Definition 2. A player $i$ is rational at state $\omega$ if he chooses 'accept' when offered to participate in a bet $F$ if and only if $E_{i} f^{i}(\omega)>0$. There is common knowledge of rationality if the event 'every player is rational' is a common knowledge event.

Definition 3. A bet $F$ is an agreeable bet if there is common knowledge of rationality, and common knowledge that all players accept the bet.

Given the previous definitions, another way of stating that a bet is agreeable at $\omega$ is to stipulate that $E_{\mu^{i}} f^{i}\left(\omega^{\prime}\right)>0$ for all $i$ and all states $\omega^{\prime}$ contained in the meet element $\Pi(\omega)$. This is the way it is usually presented in the literature. The implication is that all players believe they have positive expectations of winning the bet, and that this is common knowledge, even though the bet is zero sum in every possible state of the world.

In the special case of a two-player type space Definition 3 implies that we may consider a random variable $f$ to be an agreeable bet if it is common knowledge that $E_{\mu^{1}} f>0>E_{\mu^{2}} f$ (by working with the pair $\{f,-f\}$ ).

We can now state the Aumann No Disagreement Theorem:

A compact type space has a common prior if and only if there does not exist an agreeable bet.

The most accessible proof of this result is in Heifetz (2006). The seminal work on no disagreement is the famous paper of Aumann (1976), which proved the result in one direction (a common prior implies no disagreement) in the special case of finite type spaces and bets that are restricted to characteristic functions over events. This was extended to bets that are any random variable over a finite type space with a common prior by Sebenius and Geanakoplos (1983). The converse direction was independently proved by Morris (1995) and Samet (1998) for finite type spaces and by Feinberg (2000) for compact type spaces.

Weakening the condition that the posterior values players assign to events are common knowledge, Monderer and Samet (1989) extend the no disagreement theorem by showing that if there is a common prior and common $p$-belief regarding the posteriors players assign to an event then those posterior values cannot differ by more than $2(1-p)$. Following this, Neeman (1996a) showed that this upper bound on disagreement can be reduced to $1-p$ (but no further).

\section{BOUNDED DISAGREEMENTS}

Given the negative result of the previous section, a natural question that arises is what happens when the assumptions of common priors and common knowledge of a particular event (namely, the event of positive expected 
values on the part of all players) are weakened. This is what we will do in the remaining of this paper.

The established measure of how far players are from common knowledge of an event is the concept of common $p$ belief, introduced by Monderer and Samet (1989). We present in Section 4.2 a way to measure how far a type space is from having a common prior. In Section 4.3 we put these together common $p$-belief and our measure of the distance from common prior to study bounds on possible disagreements between players when both the common prior and the common knowledge assumptions are weakened.

\subsection{Background Assumptions.}

We assume throughout a fixed two-player type space $\mathcal{T}$ with each the partition $\Pi^{i}$ for each player $i$ further assumed to be finite, such that $\mathcal{T}$ is connected, i.e., $\Pi^{1} \wedge \Pi^{2}=\{\Omega\}$. This enables us to equate statements regarding common knowledge of an event with statements regarding mutual knowledge of the event at every state in $\Omega$, simplifying the presentation. The generalisation to non-connected spaces, which can always be decomposed to connected subspaces, is straight-forward.

Given a distribution $\mu \in \Delta(\Omega)$ and an event $A$, if $A \cap \operatorname{supp}(\mu)=\emptyset$, then $E_{\mu}(f \mid A)$ in (1) is undefined. It turns out, however, that for our purposes in this paper, we may conveniently adopt the convention that

$$
E_{\mu}(f \mid A)= \begin{cases}\frac{1}{\mu(A)} \int_{\omega \in A} f(\omega) d \mu & \text { if } \mu(A) \neq 0 \\ 0 & \text { if } \mu(A)=0\end{cases}
$$

This convention will be assumed throughout the rest of this paper.

\subsection{Prior Distance.}

The main idea here is straightforward. Each player has a compact and closed set of priors. When these sets intersect - intuitively speaking, when there is 'zero distance' between them - there is a common prior. Thus, if they are disjoint, we will seek points in player 1's set of priors and points in player 2's set of priors, such that the distance between these points is the 'smallest distance between the sets of priors'. Since we are trying to find bounds on disagreements, the 'distance' needs to measure how 'far apart' distributions are with regard to conditional probabilities, given an event. To capture notions of closeness of priors, we need a topology.

Given $\varphi^{1}, \varphi^{2} \in \Delta(\Omega)$, a random variable $f$, and an event $A \in \Sigma$, define $d^{\prime}$ by:

$$
d^{\prime}\left(\varphi^{1}, \varphi^{2}, A, f\right):=\left|E_{\varphi^{1}}(f \mid A)-E_{\varphi^{2}}(f \mid A)\right| .
$$

In words, $d^{\prime}$ is the absolute difference between the expectations assigned by $\varphi^{1}$ and $\varphi^{2}$ to $f$ conditional on $A$. 
Denote by $B$ the collection of measurable random variables $f$ over $\Omega$ satisfying the property that $0 \leq f(\omega) \leq 1$ for all $\omega \in \Omega$, and then use $d^{\prime}$ to define

$$
d^{\prime \prime}\left(\varphi^{1}, \varphi^{2}, A\right):=\sup _{f \in B} d^{\prime}\left(\varphi^{1}, \varphi^{2}, A, f\right) .
$$

Restricting the random variables to the closed and bounded set $B$ ensures that the supremum in Equation (7) is a well-defined real number.

Finally, define

$$
d\left(\varphi^{1}, \varphi^{2}\right):=\sup _{A \in \Sigma} d^{\prime \prime}\left(\varphi^{1}, \varphi^{2}, A\right) .
$$

Lemma 1, in the appendix, shows that $d$ in fact defines a metric over $\Delta(\Omega){ }^{4}$

Intuitively, $d$ measures the 'worst case' of disagreement between agents holding beliefs $\varphi^{1}$ and $\varphi^{2}$ (over all normalised random variables). By the normalisation assumption, $0 \leq d\left(\varphi^{1}, \varphi^{2}\right) \leq 1$ for any pair of distributions $\varphi^{1}$ and $\varphi^{2} \in \Delta(\Omega)$.

Note also the following: suppose that there is an event $A$ such that $\varphi^{i}(A)=$ 0 and $\varphi^{j}(A) \neq 0$. Then, using Equation (5) and setting $f$ to be $f(\omega)=1$ for all $\omega \in A$, we have $E_{\varphi^{i}}(f \mid A)=0$ while $E_{\varphi^{j}}(f \mid A)=1$, so that $d^{\prime \prime}\left(\varphi^{1}, \varphi^{2}, A\right)=1$, and therefore $d\left(\varphi^{1}, \varphi^{2}\right)=1$. This makes perfect intuitive sense: if $\varphi^{i}(A)=0$ and $\varphi^{j}(A) \neq 0$, then $i$ and $j$ have the most 'violent disagreement' possible - one regards the expected value of any $f$ given $A$ to be a well-defined real number, the other regards it as undefined, because he regards $A$ as impossible. Hence $\varphi^{1}$ and $\varphi^{2}$ should being maximally distant under $d$, which is exactly the intuitive interpretation we give to $d\left(\varphi^{1}, \varphi^{2}\right)=1$.

Definition 4. Given a type space $\mathcal{T}$, and the related sets of priors $W^{1}$ and $W^{2}$ of players 1 and 2 respectively, the value

$$
\delta=\inf _{\varphi \in W^{1}, \varphi^{\prime} \in W^{2}} d\left(\varphi, \varphi^{\prime}\right)
$$

will be called the prior distance of $\mathcal{T}$. A pair of priors $\mu^{1} \in W^{1}$ and $\mu^{2} \in W^{2}$ such that $d\left(\mu^{1}, \mu^{2}\right)=\delta$ are a pair of $\delta$-nearest priors of $\mathcal{T}$.

Definition 4 intuitively measures the distance between the pair of sets of priors of players 1 and $2, W^{1}$ and $W^{2}$, by identifying the 'closest points' between the two sets according to the metric $d$. If the sets have non-empty intersection, there is a common prior (hence no disagreement) and the prior distance is zero. If the prior distance $\delta$ is non-zero, the sets of priors are non-intersecting, there is no common prior (hence there is disagreement),

\footnotetext{
4 This metric induces a topology on the space of measures $\Delta(\Omega)$, and the resulting topology is finer than the usual weak* topology.
} 
and $\delta$ can be interpreted as providing a measure of possible posterior disagreement.

\subsection{Almost-Almost: Almost Common Knowledge with Almost Com- mon Priors.}

Monderer and Samet (1989) consider models with common priors but 'almost common knowledge', via common $p$-beliefs. With the notion of nearest priors, we can relax both the assumption of common priors and the assumption of common knowledge, to yield an 'almost-almost' result, with nearest priors serving as a proxy for 'almost common priors', and common $p$-beliefs as 'almost common knowledge', bounding disagreements regarding posteriors.

Let $\mathcal{T}$ be a connected type space. Suppose that $\mathcal{T}$ has $\delta$-prior distance, with $\left(\mu^{1}, \mu^{2}\right)$ a pair of $\delta$-nearest priors. Fix a random variable $g$ with $0 \leq$ $g(\omega) \leq 1$ for all $\omega$.

Next, define functions $f^{i}$, for the players $i=1,2$, by

$$
f^{i}(\omega):=\mu^{i}\left(g \mid \Pi^{i}(\omega)\right) ;
$$

i.e., $f^{i}(\omega)=f^{i}\left(\Pi^{i}(\omega)\right)$ is $i$ 's posterior probability of $g$, given $\Pi^{i}(\omega)$. Let $\eta_{i}$ be two numbers in the interval $[0,1]$, satisfying $\eta_{1} \geq \eta_{2}$. Consider the event

$$
C=\left\{\omega \mid f^{1}(\omega) \geq \eta_{1}\right\} \cap\left\{\omega \mid f^{2}(\omega) \leq \eta_{2}\right\} .
$$

In words, $C$ is the event that player 1 's posterior probability of $g$ is greater than or equal to $\eta_{1}$ and player 2's posterior probability of $g$ is less than or equal to $\eta_{2}$.

Proposition 1. Suppose that $\mathcal{T}$ has $\delta$-prior distance and that there is common p-belief at a state $\omega^{*}$ in the event

$$
C=\left\{\omega \mid f^{1}(\omega) \geq \eta_{1}\right\} \cap\left\{\omega \mid f^{2}(\omega) \leq \eta_{2}\right\} .
$$

Then $\left|\eta_{1}-\eta_{2}\right| \leq 1-p(1-\delta)$.

Proposition 1 immediately leads to the following theorem:

Theorem 1. Suppose that $\mathcal{T}$ has $\delta$-prior distance and that the posteriors of an event $H$ are common p-belief at some $\omega \in \Omega$. Then those posterior beliefs cannot differ by more than $1-p(1-\delta)$.

The above result highlights how common $p$-belief and $\delta$-nearest priors work together to bound disagreements, beyond the common prior and common knowledge cases.

When $\delta=0$, i.e., there is a common prior, Theorem 1 recapitulates the result of the main theorem of Neeman (1996a) (for a pair of players), and 
when both $p=1$ and $\delta=0$, i.e., there is both a common prior and common knowledge, we recapitulate the No Disagreements Theorem of Aumann (1976). 


\section{Heterogeneous Priors and No Trade}

5.1. The Exchange Economy. Based mainly on models presented in Milgrom and Stokey (1982) and Neeman (1996b), we consider an economy with a set consisting of a pair of players $I=\{1,2\}$, operating in an environment of uncertainty represented by a type space $\mathcal{T}$. Each player $i$ has a risk neutral von-Neumann-Morgenstern utility function $u^{i}: \mathbb{R} \rightarrow \mathbb{R}$, and a prior distribution $\mu^{i}$ over $\Omega$.

For simplicity of exposition, the model here is restricted to a one-dimensional commodity space. Hence initial allocations are measured as real numbers, representing the quantity of this single commodity with which a player is endowed. Similarly a trade is an agreement to transfer a certain quantity of the commodity from one player to another.

Allocations are presumed to be state-contingent:

Definition 5. An initial allocation is assignment $e^{i}: \Omega \rightarrow \mathbb{R}_{+}$associated with each $i$. Denote by $e=\left\{e^{1}, e^{2}\right\}$ the total (initial) allocation.

Trade is similarly defined.

Definition 6. A trade $B$ between the players is a state-contingent commodity transfer, i.e., a random variable $B: \Omega \rightarrow \mathbb{R}_{+}$.

A trade is paired with a trade price $q \in \mathbb{R}_{+}$. If a trade at price $q$ is implemented at state $\omega$, then one of the players, $i$, buys the trade at price $q$ and the other player, $j$, sells at price $q$. This then results in a re-allocation: $e^{i}(\omega)+B(\omega)-q$ for the buyer; $e^{j}(\omega)-B(\omega)+q$ for the seller.

A feasible trade must respect the constraint that $e^{j}(\omega)-B(\omega) \geq 0$ for the seller at each state $\omega \in \Omega$.

Definition 7. Given a pair of priors $\mu^{1}, \mu^{2}$ of players 1 and 2 respectively, an allocation $e=\left\{e^{1}, e^{2}\right\}$ is ex-ante Pareto optimal if there does not exist a feasible trade $B$ with price $q$ such that

$$
E_{\mu^{i}}\left(u^{i}\left(e^{i}+B-q\right)\right) \geq E_{\mu^{i}}\left(u^{i}\left(e^{i}\right)\right)
$$

for the buyer $i$ and

$$
E_{\mu^{j}}\left(u^{j}\left(e^{j}-B+q\right)\right) \geq E_{\mu^{j}}\left(u^{j}\left(e^{j}\right)\right)
$$

for the seller $j$ with strict inequality for at least one of $i$ or $j$.

The state contingency inherent in the definition of feasible trade in Definition 6, and the ex ante aspect of Definition 7 require some comment here. Conceptually, in the ex ante stage, before the players know the true state or have even received any signals regarding the identity of the true state, they are contemplating a commodity transfer that will occur when the true state 
is revealed. This state of affairs may be possible if there exists a complete set of Arrow-Debreu securities, so that trades contingent on the true state of the world are possible.

5.2. Rational Trade. As in the model of betting above, players offered a trade and a trade price may choose from a set of available actions

$$
A=\{\text { 'buy', 'sell', 'refrain' }\} \text {. }
$$

A player who at state $\omega$ buys a trade $B$ at price $q$ pays $q$ and therefore receives $e^{i}(\omega)+B(\omega)-q$ if the true state is $\omega$; the seller receives $q$ and gives $B(\omega)$

We denote by $s^{i}(\omega, B, q)$ player $i$ 's strategy function. By this we mean that for each observed partition $\Pi^{i}(\omega)$, trade $B(\omega)$ that is offered, and trade price $q$ that is suggested, the strategy function of trader $i$ prescribes an action in $A$. The collection of strategy functions of player $i$ is denoted by $S^{i}$ A strategy profile is a pair $\left(s^{i}, s^{j}\right)$ of strategy functions, for $i \neq j$. The collection of all strategy profiles is denoted as $S$, with the generic element written as $s=\left(s^{1}, s^{2}\right)$.

In choosing his strategy, trader $i$ takes into account his utility function $u^{i}$ for money. Given that a trade occurs if and only if one trader is willing to sell and the other to buy, we can derive a state-contingent 'utility function from trade' (as opposed to utility from money) for each trader $i$, labelled $v^{i}\left(s^{i}(\omega), s^{j}(\omega), B, q\right)$ as

$v^{i}(s(\omega), B, q)= \begin{cases}u^{i}\left(e^{i}(\omega)+B(\omega)-q\right) & \text { if } s^{i}(\omega)=\text { 'buy', } s^{j}(\omega)=\text { 'sell' } \\ u^{i}\left(e^{i}(\omega)-B(\omega)+q\right) & \text { if } s^{i}(\omega)=\text { 'sell', } s^{j}(\omega)=\text { 'buy' } \\ u^{i}\left(e^{i}(\omega)\right) & \text { otherwise. }\end{cases}$

Definition 8. Player $i$ is rational at state $\omega$ under strategy profile $s=\left(s^{i}, s^{j}\right)$ with respect to a proposed feasible trade $B$ and price $q$ if

$$
s^{i}(\omega, B, q) \in \arg \max _{s^{i} \in S^{i}} E_{i}\left(v^{i}\left(s^{i}(\omega), s^{j}(\omega), B, q\right) \mid \Pi^{i}(\omega)\right) .
$$

There is common knowledge of rationality under a strategy profile if the event that both players are rational is common knowledge.

There is positive probability of trade if the event $Z:=\left\{\omega \mid s^{i}(\omega, B, q)=\right.$ 'buy', $s^{j}(\omega, B, q)=$ 'sell' $\}$ satisfies $\mu^{i}(Z)>0$ and $\mu^{j}(Z)>0$.

Milgrom and Stokey (1982) proved what has come to be called the No Trade theorem: in a knowledge structure with a common prior and ex ante Pareto efficiency, rational agents can never agree to a commonly known and mutually acceptable feasible trade in the interim period after they receive private information and calculate their posterior expected values. (To be precise, the result in Milgrom and Stokey (1982) supposes the weaker 
condition of concordant beliefs. However, the existence of a common prior implies concordant beliefs, and the No Trade Theorem is widely cited as holding under the common prior assumption.)

Note that the statement of the theorem goes in only one direction: a common prior and common knowledge of acceptance of a trade nullifies a possibility of trade occurring. In contrast, the no disagreements theorem, as extended to no betting, is bi-directional: if there is a common prior there is no betting, and if there is no common prior then there exists a mutually agreeable bet.

It is tempting to try to conclude that no trade can similarly be extended to its converse, that is, that if there is no common prior then traders will able in the interim period to conduct a mutually agreeable trade. This is because, if one assumes that $e^{1}(\omega)=e^{2}(\omega)=0$ uniformly, then given a proposed trade $B$ with price $q$, one may form a profile of random variables

$$
f^{i}(\omega):=B(\omega)-q
$$

for the buyer and

$$
f^{j}(\omega):=B(\omega)+q
$$

for the seller. It then appears as if the existence of an agreed trade is equivalent to an agreeable bet $F=\left\{f^{1}, f^{2}\right\}$, and one is guaranteed the existence of such a bet when priors are not common.

The difference between No Betting and No Trade, however, is that in the latter the condition of ex-ante Pareto efficiency must be satisfied, whereas there is no such requirement for the former. That, as is shown in the next section, makes all the difference: even when the priors are not common, No Trade holds.

\subsection{No Trade.}

Theorem 2. For a pair of players, let $u^{1}$ and $u^{2}$ respectively be a pair of (not necessarily risk-neutral nor increasing) utility functions, $\mu^{1}$ and $\mu^{2}$ respectively be a pair of priors (allowing for heterogeneous priors), and $e^{1}$ and $e^{2}$ respectively be a pair of allocations that are ex-ante Pareto-efficient with respect to $\mu^{1}$ and $\mu^{2}$.

Suppose that there exists a trade $B$, a price $q$, and a pair of strategies $s^{1}, s^{2}$ such that there is common knowledge of rationality and common knowledge of a positive probability of trade. Then neither player can strictly prefer trade to no-trade.

The result of Theorem 2 strengthens those of Milgrom and Stokey (1982), Dow et al (1990), and even Neeman (1996b), since it allows for the mere 
existence of positive probability of trade and does not require common priors.

We again note here that the difference between the no trade result of Theorem 2 and the no betting theorem comes down to the assumption of ex ante Pareto efficiency in the no trade result, which plays no role in the no betting result. To see this, consider running the argument of Theorem 2 with respect to bets instead of trades.

The essence of the original proof of Aumann (1976) that 'common priors imply no agreement' is rather similar to the heart of the proof of Theorem 2, namely any common knowledge disagreement that holds in each partition element can be aggregated to a disagreement over the entire state space, contradicting the assumption of a common prior. If there are heterogenous priors, one could run exactly the same argument. The result would be nonequal ex ante expected values, one per each player, but that would not lead to any contradiction, since heterogeneous priors can accommodate ex ante disagreement regarding bets in the full state space. In contrast, in the trade model, any mutually accepted interim trade (under common knowledge) is translated under aggregation into a mutually accepted $e x$ ante trade, violating the ex ante Pareto efficiency assumption even when there are heterogeneous priors.

\section{Heterogeneous Priors and Trade}

\subsection{Rational Strategies.}

Rationality of a player offered a trade at a state $\omega$ was defined in Definition 8 . Denote by $R^{i}\left(B, q, s^{j}, \omega\right)$ the collection of all strategies of player $i$ that are rational at state $\omega$ with respect to a fixed strategy $s^{j}$ and a given $B$ and $q$.

As in games with incomplete information, rationality is defined interactively, as being trader $i$ 's best response to $s^{j}$. Given $B$, $q$, and a fixed strategy function $s^{j}$ of player $j$, let $R^{i}\left(B, q, s_{j}\right)$ denote the collection of strategy functions of player $i$ that are rational with respect to $s^{j}$ at all states, i.e.,

$$
R^{i}\left(B, q, s_{j}\right):=\left\{s^{i} \mid s^{i} \in R^{i}\left(B, q, s_{j}, \omega\right), \forall \omega \in \Omega\right\} .
$$

Assuming w.l.o.g. that trader 1 is the buyer and trader 2 is the seller, let $Z(s, B, q)$ denote the event at which both traders are rational and trade is beneficial to both parties:

$$
\begin{aligned}
& Z(s, B, q) \\
& :=\left\{\omega \in \Omega \mid \begin{array}{ll}
\text { 'buy' }=\arg \max E\left(v^{1}(s(\omega), B, q) \mid \Pi^{1}(\omega)\right) \\
\text { 'sell' }=\arg \max E\left(v^{2}(s(\omega), B, q) \mid \Pi^{2}(\omega)\right)
\end{array}\right\}
\end{aligned}
$$


As Theorem 2 indicates, for trade to be feasible it is insufficient to weaken the common prior assumption. In fact, one needs also to weaken the assumption of common knowledge of rationality; that is, there needs to be a 'suspicion of irrationality' in the sense that each trader believes he is rational but suspects that the other trader is not.

Making this precise requires adopting a bounded rationality approach. There are several models of bounded rationality in the literature. We will make use here of the minimal bounded rationality assumption necessary for trade to occur in the context of heterogeneous priors. Specifically, we let common knowledge of rationality be weakened to common $p$-belief in rationality for $p$ arbitrarily close to 1 , where furthermore each agent believes himself to be rational with probability 1 .

\subsection{Common $p$-Belief in Rationality and Trade.}

Neeman (1996b) presents a model of common $p$-belief in rationality, in the context of common priors, that enables trade to occur with unbounded volumes of trade. For that model to work, each trader must assume that there is a non-zero probability that he/she himself/herself may be irrational. There are interpretational difficulties in such an approach; noting this, in the concluding remarks of that paper, Neeman (1996b) states that 'it might be argued that using different priors alleviates some of the difficulties with the interpretation of the model, in particular regarding the way we chose to model irrationality'.

We show here in detail in Theorem 3 that this is indeed true: in a model of heterogeneous priors, common $p$-belief in rationality enables trade to occur under conditions of 'suspicion of irrationality', in which each trader asserts zero probability that he is irrational but ascribes positive probability that the other trader is irrational. In addition, although the volume of trade may be unlimited, by measuring how far the traders are from a common prior using prior distance, Theorem 3 provides limits on the range of prices under which trade may occur.

The next definition, of $\left(1-\rho^{1}, 1-\rho^{2}\right)$-rationality Interim Nash Equilibrium, is inspired by a similarly named definition in Neeman (1996b) (which supposes a common prior). Here we allow for $\mu^{i} \neq \mu^{j}$.

Definition 9. A pair of strategies $s^{1}(B, q)$ and $s^{2}(B, q)$ is a $\left(1-\rho^{1}, 1-\rho^{2}\right)$ rationality Interim Nash Equilibrium, for $0 \leq \rho^{1}, \rho^{2}<1$, if there exists another pair of strategies $\bar{s}^{1}(B, q)$ and $\bar{s}^{2}(B, q)$ such that for each $i$ and $j$, $i \neq j$,

(1) there exists an event $F^{j} \subset \Omega$ such that $\mu^{i}\left(F^{j}\right) \leq \rho^{i}$

(2) for all $\omega \in \Omega \backslash F^{1} \cup F^{2}$, one has $s^{i} \in R^{i}\left(B, q, s^{j}, \omega\right)$ 
(3) for all $\omega \in F^{i}$, one has
(a) $s^{j} \in R^{j}\left(B, q, \bar{s}^{i}, \omega\right)$,
(b) $\bar{s}^{i} \notin R^{i}\left(B, q, s^{j}, \omega\right)$

In words, each player $i$ believes that he or she is rational, because for $\omega \in$ $\Omega \backslash F^{j}$, for each $i$ and $j$, there exists a set of states $F^{j}$ such that each trader $i$ ascribes probability $\rho^{i}$ to this set and at which trader $j$ chooses a suboptimal strategy to play to which playing strategy $s^{i}(\cdot)$ constitutes a best reply from the side of player $i$. It is in this sense that (erroneous) belief in irrationality enters the model, with each player mistaking the rationality of the other player.

Definition 10 is taken directly from Neeman (1996b).

Definition 10. Let $0<p<1$. Then two partitions $\Pi^{1}$ and $\Pi^{2}$ are $p$ overlapping if for each trader $i$ there exists an index set $K^{i}$ and a non-empty set $\pi^{i}=\bigcup_{k \in K^{i}} \Pi_{k}^{i}$ such that the following two conditions hold:

(1) $\mu^{i}\left(\pi^{j} \mid \Pi_{k}^{i}\right) \geq p$ for all $\Pi_{k}^{i} \subseteq \pi^{i}$; and

(2) for any two non-empty index subsets $K^{1^{\prime}} \subseteq K^{1}$ and $K^{2^{\prime}} \subseteq K^{2}$, $\mu^{i}\left(\pi^{1^{\prime}} \Delta \pi^{2^{\prime}}\right)>0$ for at least one $i$, where $\pi^{i^{\prime}}=\bigcup_{k \in K^{i^{\prime}}} \prod_{k}^{i}$ and $\Delta$ denotes symmetric difference.

The definition of $p$-overlapping partitions is quite technical but it essentially provides a necessary and sufficient condition for the existence of a common $p$-belief event, namely $\pi^{1} \cap \pi^{2}$, that is not common knowledge, nor are any of it sub-events common knowledge either. The irrationality that the players ascribe to one another is then concentrated outside $\pi^{1} \cap \pi^{2}$.

Theorem 3. Let $\mathcal{T}$ be a type space with $\delta$ prior distance, with $\delta>0$. Let $\rho^{1}, \rho^{2} \geq 0$, with $\rho^{i}>0$ for at least one $i$, and let $0<p<1$. Then the following two conditions are equivalent:

(I) The information structures are p-overlapping.

(II) There exists a proposed trade B, a price q, and strategies $s^{1}(\omega), s^{2}(\omega)$ such that:

(a) the strategies $s^{1}(\omega), s^{2}(\omega)$ form a $\left(1-\rho^{1}, 1-\rho^{2}\right)$-rationality interim Nash equilibrium with respect to the trade $(B, q)$.

(b) Rationality is heterogeneous common p-belief at some $\omega \in \Omega$.

Furthermore, the range of the price $q$ at which trade may occur is limited by $y^{1}>q>y^{2}$, where $y^{1}-y^{2} \leq(1-p(1-\delta))\|B\|_{\infty}$.

Proof. See Appendix.

As Theorem 3 indicates, for trade to occur it suffices for one trader to ascribe positive probability to the other trader being irrational (not necessarily 
both traders), while each trader ascribes zero probability to himself being irrational.

\subsection{Common $p$-Belief in Rationality: The General Case.}

In this subsection we allow the functions $u_{i}$ to incorporate agents' risk aversion. Utility functions are parametrised by two positive parameters $M, \psi$. More specifically, we consider the set of strictly increasing, concave, and differentiable utility functions

$$
U_{M, \psi}=\left\{u:[-M, M] \rightarrow \mathbb{R} \mid u^{\prime}(M) \geq \psi\right\},
$$

allowing for a general preference representation. It turns out that the previous theorem extends easily to this more general case, provided that agents are not too risk averse, in which case one can find a non-empty range of the prices allowing for the trade to occur. To parametrize the range of prices, it is useful to introduce the following notation. Define $C^{i}\left(\mu^{i}, u^{i}\right)$ as

$$
C^{i}\left(\mu^{i}, u^{i}\right):=\min _{\Pi_{k}^{i}(\omega) \in \pi^{i}} \hat{C}^{i}\left(\mu^{i}, u^{i}, \Pi_{k}^{i}(\omega)\right)
$$

where $\hat{C}^{i}\left(\mu^{i}, u^{i}, \Pi_{k}^{i}(\omega)\right)$ is a positive number given by

$$
\begin{aligned}
E_{i}\left(u ^ { i } \left(e^{i}(\omega)+(-1)^{i-1}\left(B(\omega)-\hat{C}^{i}\left(\mu^{i}, u^{i}, \Pi_{k}^{i}(\omega)\right)\right) \mid\right.\right. & \left.\Pi_{k}^{i}(\omega)\right) \\
& =E_{i}\left(u^{i}\left(e^{i}(\omega)\right) \mid \Pi_{k}^{i}(\omega)\right)
\end{aligned}
$$

for all $\left.\Pi_{k}^{i}(\omega)\right) \in \pi^{i}$.

Theorem 4. Let $\mathcal{T}$ be a type space with $\delta$ prior distance, with $\delta>0$. Let $\rho^{1}, \rho^{2} \geq 0$, with $\rho^{i}>0$ for at least one $i$, and $p<1$. Let positive $\psi$ and $M$ be arbitrarily given. Then the following two conditions are equivalent:

(I) There exists a $\sigma>0$ such that there exists a trade $B$, a price $q$, and strategies $s^{1}(\omega), s^{2}(\omega)$ such that for all traders with utility functions $u \in U_{M, \psi}$ that satisfy $\sup _{|x|<M}\left|u^{\prime \prime}(x)\right|<\sigma$ (i.e. sufficiently risk tolerant traders):

(a) the strategies $s^{1}(\omega), s^{2}(\omega)$ form a $\left(1-\rho^{1}, 1-\rho^{2}\right)$-rationality interim Nash equilibrium with respect to the trade $(B, q)$.

(b) Rationality is heterogeneous common p-belief at some $\omega \in \Omega$.

(II) The information structures are p-overlapping.

Furthermore, the range of prices $q$ at which trade may occur is limited by $y^{1}-C^{1}\left(\mu^{1}, u^{1}\right)>q>y^{2}+C^{2}\left(\mu^{2}, u^{2}\right)$ with $y^{1}-y^{2} \leq(1-p(1-\delta))\|B\|_{\infty}-$ $\left(C^{1}\left(\mu^{1}, u^{1}\right)+C^{2}\left(\mu^{2}, u^{2}\right)\right)$. 
Hence, compared to the (risk neutral) result of Theorem 3, the more risk averse the agents are (i.e. the higher are $C^{1}\left(\mu^{1}, u^{1}\right)$ and $\left.C^{2}\left(\mu^{2}, u^{2}\right)\right)$, ${ }^{5}$ the smaller is the range of prices under which trade will happen.

\section{CONCLUding REMARKS}

In this paper we have shown that on one hand, the No Trade theorem obtains even if players do not share a common prior; on the other hand a small departure from the common knowledge of rationality is sufficient to restore the positive trade result. One possible interpretation is that the player involved exhibit over-confidence, trusting themselves to be fully rational but suspecting that others may sometimes be irrational or incorrectly interpreting signals.

The perceived departure from full common knowledge of rationality may be arbitrarily small, i.e. in common $p$-belief of rationality $p$ can approach 1 arbitrarily close (provided that information structures remain $p$-overlapping) and moreover each agent can believe himself to be perfectly rational. In this sense, it suffices that at least one agent $i$ ascribe a small probability that at some state player $j$ erroneously trades where he or she should not trade (as trade there does not maximise player's $j$ expected utility).

\section{APPENDIX - PROOFS}

Lemma 1. $d$ is a metric.

Proof. By definition, $d\left(\varphi^{1}, \varphi^{2}\right) \geq 0$ for all $\varphi^{1}, \varphi^{2} \in \Delta(\Omega)$, and it is almost immediately clear that equality holds if and only if $\varphi^{1}=\varphi^{2}$.

Similarly, that $d\left(\varphi^{1}, \varphi^{2}\right)=d\left(\varphi^{2}, \varphi^{1}\right)$ follows from the definition.

For the triangle inequality, suppose $\varphi^{1}, \varphi^{2}, \varphi^{3} \in \Delta(\Omega)$. Temporarily fix $A \in \Sigma$ and $f \in B$. We initially want to establish

$$
d^{\prime}\left(\varphi^{1}, \varphi^{3}, A, f\right) \leq d^{\prime}\left(\varphi^{1}, \varphi^{2}, A, f\right)+d^{\prime}\left(\varphi^{2}, \varphi^{3}, A, f\right),
$$

which holds true in all possible cases, as we detail:

(a) If $A \cap \operatorname{supp}\left(\varphi^{1}\right)=\emptyset$ and $A \cap \operatorname{supp}\left(\varphi^{3}\right)=\emptyset$ :

$d^{\prime}\left(\varphi^{1}, \varphi^{3}, A, f\right)=0$, and Equation (11) is automatically satisfied.

(b) If $A \cap \operatorname{supp}\left(\varphi^{1}\right)=\emptyset, A \cap \operatorname{supp}\left(\varphi^{2}\right)=\emptyset$ and $A \cap \operatorname{supp}\left(\varphi^{3}\right) \neq \emptyset$ :

$d^{\prime}\left(\varphi^{1}, \varphi^{3}, A, f\right)=d^{\prime}\left(\varphi^{2}, \varphi^{3}, A, f\right)=\left|E_{\varphi^{3}}(f \mid A)\right|$ and $d^{\prime}\left(\varphi^{1}, \varphi^{2}, A, f\right)=$

0 . Then $d^{\prime}\left(\varphi^{1}, \varphi^{3}, A, f\right)=d^{\prime}\left(\varphi^{1}, \varphi^{2}, A, f\right)+d^{\prime}\left(\varphi^{2}, \varphi^{3}, A, f\right)$.

\footnotetext{
${ }^{5}$ Here $C^{i}\left(\mu^{i}, u^{i}\right)$ corresponds to the amount needed to compensate player $i$ for undertaking the risk of the bet, making him indifferent between taking or rejecting the bet. Note that $C^{i}\left(\mu^{i}, u^{i}\right)$ depends on $B(\omega)$.
} 
(c) If $A \cap \operatorname{supp}\left(\varphi^{1}\right)=\emptyset, A \cap \operatorname{supp}\left(\varphi^{2}\right) \neq \emptyset$ and $A \cap \operatorname{supp}\left(\varphi^{3}\right) \neq \emptyset$ :

$d^{\prime}\left(\varphi^{2}, \varphi^{3}, A, f\right)=\left|E_{\varphi^{2}}(f \mid A)-E_{\varphi^{3}}(f \mid A)\right|, d^{\prime}\left(\varphi^{1}, \varphi^{3}, A, f\right)=$ $\left|E_{\varphi^{3}}(f \mid A)\right|$, and $d^{\prime}\left(\varphi^{1}, \varphi^{2}, A, f\right)=\left|E_{\varphi^{2}}(f \mid A)\right|$. Since $E_{\varphi^{3}}(f \mid A)=$ $E_{\varphi^{2}}(f \mid A)-E_{\varphi^{2}}(f \mid A)-E_{\varphi^{3}}(f \mid A)$, it follows that $d^{\prime}\left(\varphi^{1}, \varphi^{3}, A, f\right) \leq$ $d^{\prime}\left(\varphi^{1}, \varphi^{2}, A, f\right)-d^{\prime}\left(\varphi^{2}, \varphi^{3}, A, f\right)$.

(d) If $A \cap \operatorname{supp}\left(\varphi^{1}\right) \neq \emptyset, A \cap \operatorname{supp}\left(\varphi^{2}\right)=\emptyset$ and $A \cap \operatorname{supp}\left(\varphi^{3}\right)=\emptyset$ : $d^{\prime}\left(\varphi^{1}, \varphi^{3}, A, f\right)=d^{\prime}\left(\varphi^{1}, \varphi^{2}, A, f\right)=\left|E_{\varphi^{1}}(f \mid A)\right|$, and $d^{\prime}\left(\varphi^{2}, \varphi^{3}, A, f\right)=$ 0 , so (11) is satisfied, since $\left|E_{\varphi^{1}}(f \mid A)\right|=\left|E_{\varphi^{1}}(f \mid A)\right|+0$.

(e) If $A \cap \operatorname{supp}\left(\varphi^{1}\right) \neq \emptyset, A \cap \operatorname{supp}\left(\varphi^{2}\right)=\emptyset$, and $A \cap \operatorname{supp}\left(\varphi^{3}\right) \neq \emptyset$ : $d^{\prime}\left(\varphi^{1}, \varphi^{3}, A, f\right)=\left|E_{\varphi^{1}}(f \mid A)-E_{\varphi^{3}}(f \mid A)\right|, d^{\prime}\left(\varphi^{1}, \varphi^{2}, A, f\right)=$ $\left|E_{\varphi^{1}}(f \mid A)\right|$, and $d^{\prime}\left(\varphi^{2}, \varphi^{3}, A, f\right)=\left|E_{\varphi^{3}}(f \mid A)\right|$. Since

$$
\left|E_{\varphi^{1}}(f \mid A)-E_{\varphi^{3}}(f \mid A)\right| \leq\left|E_{\varphi^{1}}(f \mid A)\right|+\left|E_{\varphi^{3}}(f \mid A)\right|,
$$

(11) is satisfied.

(f) If $A \cap \operatorname{supp}\left(\varphi^{1}\right) \neq \emptyset, A \cap \operatorname{supp}\left(\varphi^{2}\right) \neq \emptyset$ and $A \cap \operatorname{supp}\left(\varphi^{3}\right)=\emptyset$ :

$d^{\prime}\left(\varphi^{1}, \varphi^{3}, A, f\right)=\left|E_{\varphi^{1}}(f \mid A)\right|, d^{\prime}\left(\varphi^{1}, \varphi^{2}, A, f\right)=\mid E_{\varphi^{1}}(f \mid A)-$ $E_{\varphi^{2}}(f \mid A) \mid$, and $d^{\prime}\left(\varphi^{2}, \varphi^{3}, A, f\right)=\left|E_{\varphi^{2}}(f \mid A)\right|$. Since $E_{\varphi^{1}}(f \mid A)=$ $E_{\varphi^{1}}(f \mid A)-E_{\varphi^{2}}(f \mid A)+E_{\varphi^{2}}(f \mid A)$, it follows that $\left|E_{\varphi^{1}}(f \mid A)\right| \leq$ $\left|E_{\varphi^{1}}(f \mid A)-E_{\varphi^{2}}(f \mid A)\right|+\left|E_{\varphi^{2}}(f \mid A)\right|$, so that (11) is satisfied.

(g) If $A \cap \operatorname{supp}\left(\varphi^{1}\right) \neq \emptyset, A \cap \operatorname{supp}\left(\varphi^{2}\right) \neq \emptyset$ and $A \cap \operatorname{supp}\left(\varphi^{3}\right) \neq \emptyset$ :

Since $E_{\varphi^{1}}(f \mid A)-E_{\varphi^{3}}(f \mid A)=E_{\varphi^{1}}(f \mid A)-E_{\varphi^{2}}(f \mid A)+E_{\varphi^{2}}(f \mid A)-$ $E_{\varphi^{3}}(f \mid A)$,

$\left|E_{\varphi^{1}}(f \mid A)-E_{\varphi^{3}}(f \mid A)\right| \leq\left|E_{\varphi^{1}}(f \mid A)-E_{\varphi^{2}}(f \mid A)\right|+\left|E_{\varphi^{2}}(f \mid A)-E_{\varphi^{3}}(f \mid A)\right|$

which in this case is inequality (11).

Given Equation (11), by definition of $d^{\prime \prime}, d^{\prime}\left(\varphi^{1}, \varphi^{3}, A, f\right) \leq d^{\prime \prime}\left(\varphi^{1}, \varphi^{2}, A\right)+$ $d^{\prime \prime}\left(\varphi^{2}, \varphi^{3}, A\right)$, and therefore $d^{\prime \prime}\left(\varphi^{1}, \varphi^{3}, A\right)=\sup _{f \in B} d^{\prime}\left(\varphi^{1}, \varphi^{3}, A, f\right) \leq$ $d^{\prime \prime}\left(\varphi^{1}, \varphi^{2}, A\right)+d^{\prime \prime}\left(\varphi^{2}, \varphi^{3}, A\right)$. Similar reasoning, with respect to $d^{\prime \prime}$ and varying $A \in \Sigma$, establishes that $d\left(\varphi^{1}, \varphi^{3}\right) \leq d\left(\varphi^{1}, \varphi^{2}\right)+d\left(\varphi^{2}, \varphi^{3}\right)$.

Proof of Proposition 1. If $p=0$, the conclusion is trivially true, so we can assume that $p>0$.

By assumption, $C$ is common $p$-belief at $\omega^{*}$. Then, by Equation (4), there exists an evident $p$-belief event $E \ni \omega^{*}$ such that $E \subseteq B_{i}^{p}(C)$, for all $i \in I$. Define $\pi^{1}=B_{1}^{p}(E)=\left\{\omega \in \Omega \mid \mu^{1}\left(E \mid \Pi^{1}(\omega)\right) \geq p\right\}$, $\pi^{2}=B_{2}^{p}(E)=\left\{\omega \in \Omega \mid \mu^{2}\left(E \mid \Pi^{2}(\omega)\right) \geq p\right\}$, and let $\pi=\pi^{1} \cap \pi^{2}$. The fact that $C$ is common $p$-belief at $\omega^{*}$ guarantees that $\pi$ is not empty and that $\mu^{1}(\pi)>0$, and $\mu^{2}(\pi)>0$.

Because $E$ is evident $p$-belief, by Equation (3)

(12) $E \subseteq B_{1}^{p}(E)$, and $E \subseteq B_{2}^{p}(E)$, hence $E \subseteq B_{1}^{p}(E) \cap B_{2}^{p}(E)$, 
and

$$
E \subseteq B_{1}^{p}(C) \text { and } E \subseteq B_{2}^{p}(C) .
$$

Applying monotonicity to Equation (12) yields, for $i=1,2$, that

$$
B_{i}^{p}(E) \subseteq B_{i}^{p}\left(B_{1}^{p}(E) \cap B_{2}^{p}(E)\right),
$$

or

$$
\pi^{i} \subseteq B_{i}^{p}(\pi)
$$

Applying $B_{i}^{p} B_{i}^{p}=B_{i}^{p}$ to Equation (13) yields, for $i=1,2$, that $B_{i}^{p}(E) \subseteq$ $B_{i}^{p}\left(B_{i}^{p}(C)\right)$, or

$$
\pi^{i} \subseteq B_{i}^{p}(C) .
$$

Because $B_{1}^{p}$ is measurable with respect to $\mathcal{F}^{1}, \pi^{1}$ is a union of disjoint sets $\left\{A_{k} \in \Pi^{1}\right\}$. By Equation (14), $\pi^{1} \subseteq B_{1}^{p}(\pi)$, and by definition $B_{1}^{p}(\pi)=$ $\left\{\omega \in \Omega \mid \mu^{1}\left(\pi \mid \Pi^{1}(\omega)\right) \geq p\right\}$, hence $\mu^{1}\left(\pi \mid A_{k}\right) \geq p$ for all $k$. This in turn implies that $\mu^{1}\left(\pi \mid \pi^{1}\right) \geq p$. But since $\pi \subseteq \pi^{1}$, we have that

$$
\mu^{1}\left(\pi^{2} \mid \pi^{1}\right) \geq p
$$

An exactly similar argument leads to $\mu^{2}\left(\pi^{1} \mid \pi^{2}\right) \geq p$.

We also have that $\mu^{1}\left(g \mid \pi^{1}\right)=\eta_{1}$. Otherwise, there exists an $\omega^{\prime} \in \pi^{1}$ such that $\mu^{1}\left(g \mid \Pi^{1}\left(\omega^{\prime}\right)\right) \neq \eta_{1}$, which would mean that $\mu^{1}\left(C \mid \Pi^{1}\left(\omega^{\prime}\right)\right)=0$, contradicting Equation (15). A similar argument establishes $\mu^{2}\left(g \mid \pi^{2}\right)=$ $\eta_{2}$

For any function $h$ satisfying $h \geq 0, \mu^{1}\left(h \cdot 1_{\pi^{1}}\right) \geq \mu^{1}\left(h \cdot 1_{\pi^{1}} \cdot 1_{\pi^{2}}\right)$, hence

$$
\frac{\int_{\omega \in \Omega} h(\omega) 1_{\pi^{1}}(\omega) d \mu^{1}}{\mu^{1}\left(\pi^{1}\right)} \geq \frac{\mu^{1}\left(\pi^{2} \cap \pi^{1}\right)}{\mu^{1}\left(\pi^{1}\right)} \cdot \frac{\int_{\omega \in \Omega} h(\omega) 1_{\pi^{1}}(\omega) 1_{\pi^{2}}(\omega) d \mu^{1}}{\mu^{1}\left(\pi^{1} \cap \pi^{2}\right)} .
$$

This is the same as saying $\mu^{1}\left(h \mid \pi^{1}\right) \geq \mu^{1}\left(\pi^{2} \mid \pi^{1}\right) \mu^{1}(h \mid \pi)$. Applying Equation (16) yields

$$
\mu^{1}\left(h \mid \pi^{1}\right) \geq p \mu^{1}(h \mid \pi) .
$$

Substituting $g$ for $h$ in Equation (17) gives

$$
\eta_{1} \geq p \mu^{1}(g \mid \pi) \text {. }
$$

Substituting, instead, $1-g$ for $h$ in Equation (17) yields

$$
\eta_{1} \leq p \mu^{1}(g \mid \pi)+(1-p) .
$$

We arrive at $p \mu^{1}(g \mid \pi) \leq \eta_{1} \leq p \mu^{1}(g \mid \pi)+(1-p)$. An entirely symmetric argument gives $p \mu^{2}(g \mid \pi) \leq \eta_{2} \leq p \mu^{2}(g \mid \pi)+(1-p)$. Since $\mu^{1}$ and $\mu^{2}$ are $\delta$-separated priors,

$$
\left|p \mu^{1}(g \mid \pi)-p \mu^{2}(g \mid \pi)\right|=\left|p\left(\mu^{1}(g \mid \pi)-\mu^{2}(g \mid \pi)\right)\right| \leq p \delta,
$$


and we conclude that $\left|\eta_{1}-\eta_{2}\right| \leq 1-p+p \delta$.

Proof of Theorem 1. The conditional probability of an event $H$ occurring is the conditional probability of the characteristic function $1_{H}$, hence this is a special case of Proposition 1.

Proof of Theorem 2. For trade to occur, there must exist an event

$$
Z=\left\{\omega \mid s^{i}(\omega, B, q)=\text { "buy", } s^{j}(\omega, B, q)=\text { "sell" }\right\}
$$

that both traders regard as a positive probability event, where trader $i$ buys $B$ at price $q$, and trader $j$ sells it, i.e., the associated trade is

$$
z^{i}(\omega)=(B(\omega)-q) \cdot 1_{Z}, z^{j}(\omega)=-(B(\omega)-q) \cdot 1_{Z} .
$$

The assumption of the common knowledge of rationality of the players implies that for any state $\omega$ in the connected state space $\Omega$,

$$
E_{i}\left(v^{i}\left(e^{i}(\omega)+z^{i}(\omega)\right) \mid \Pi^{i}(\omega)\right)>E_{i}\left(v^{i}\left(e^{i}(\omega)\right) \mid \Pi^{i}(\omega)\right)
$$

and

$$
E_{j}\left(v^{j}\left(e^{j}(\omega)+z^{j}(\omega)\right) \mid \Pi^{j}(\omega)\right)>E_{j}\left(v^{j}\left(e^{j}(\omega)\right) \mid \Pi^{j}(\omega)\right) .
$$

In words, Equation (20) states that at each of his interim partition elements, trader $i$ has positive expectation of beneficial trade; Equation (21) says the same with regards to trader $j$. We may now aggregate over all the partition elements of trader $i$ to the full state space $\Omega$. By the properties of the expectation operator, and following from the assumption that $\mu^{i}(Z)>0$, this yields for trader $i$ according to his prior:

$$
E_{\mu^{i}}\left(v^{i}\left(e^{i}+z^{i}\right)\right) \geq E_{\mu^{i}}\left(v^{i}\left(e^{i}\right)\right) .
$$

Doing the same for trader $j$ yields

$$
E_{\mu^{j}}\left(v^{j}\left(e^{j}+z^{j}\right)\right) \geq E_{\mu^{j}}\left(v^{j}\left(e^{j}\right)\right)
$$

Hence if even one of the traders strictly benefits from trade at event $Z$, we deduce that the initial allocations $e^{i}$ and $e^{j}$ are not ex-ante Pareto-optimal initial allocations, a contradiction.

Proposition 2. An event $C$ is heterogeneous common p-belief at $\omega \in \Omega$ if and only if for each $i \in I$ there exists an index set $K^{i}$ and a non-empty set $\pi^{i}=\bigcup_{k \in K^{i}} \Pi_{k}^{i}$ such that $\omega \in \pi^{i}$ and both of the following conditions hold:

(A1) $\mu^{i}\left(\bigcap_{h \in I} \pi^{h} \mid \Pi_{k}^{i}\right) \geq p$, for all $i \in I$ and $\Pi_{k}^{i} \subseteq \pi^{i}$

(A2) $\mu^{i}\left(C \mid \Pi_{k}^{i}\right) \geq p$, for all $i \in I$ and $\Pi_{k}^{i} \subseteq \pi^{i}$. 
Proof . $(\Rightarrow)$ Suppose that $C$ is a heterogeneous common $p$-belief at $\omega$. Then by definition there exists a heterogeneous evident $p$-belief event $E$ such that $\omega \in E$ and $E \subseteq \bigcap_{i \in I} B_{p}^{i}(C)$. Let $i \in I$ be chosen arbitrarily. Define

$$
\pi^{i}=\bigcup_{\mu^{i}\left(\pi_{k}^{i} \cap E\right)>0} \Pi_{k}^{i} .
$$

By definition, it must be the case that $E \subseteq \pi^{i}$ for all $i$, hence $E \subseteq \bigcap_{h \in I} \pi^{h}$. We also have that for all $\Pi_{k}^{i} \subseteq \pi^{i}$, because $\Pi_{k}^{i} \cap E \neq \emptyset$, there exists $\omega^{\prime} \in E$ such that $\Pi_{k}^{i}=\Pi^{i}\left(\omega^{\prime}\right)$. Hence, for all $i \in I$ and all $\Pi_{k}^{i} \subseteq \pi^{i}$

$$
\mu^{i}\left(\bigcap_{h \in I} \pi^{h} \mid \Pi_{k}^{i}\right) \geq \mu^{i}\left(E \mid \Pi_{k}^{i}\right) \geq p .
$$

By the same reasoning, $\mu^{i}\left(C \mid \Pi_{k}^{i}\right) \geq p$ for all $\Pi_{k}^{i} \subseteq \pi^{i}$.

$$
(\Leftarrow) \text { Let }
$$

$$
E=\bigcap_{h \in I} \pi^{h}
$$

Since $\omega \in \pi^{i}$ for all $i, \omega \in E$. To show that $C$ is heterogeneous common $p$-belief it suffices to show that $E$ is heterogeneous evident $p$-belief and that $E \subseteq \bigcap_{i \in I} B_{p}^{i}(C)$. Suppose that $\omega^{\prime} \in E$. By definition $\Pi^{i}\left(\omega^{\prime}\right)=\Pi_{k}^{i}$ for some $\Pi_{k}^{i} \subseteq \pi^{i}$. Since $\mu^{i}\left(\bigcap_{h \in I} \pi^{h} \mid \Pi_{k}^{i}\right) \geq p$ for every $i \in I$ and $E=\bigcap_{h \in I} \pi^{h}$, we immediately have

$$
\mu^{i}\left(E \mid \Pi^{i}\left(\omega^{\prime}\right)\right)=\mu^{i}\left(E \mid \Pi_{k}^{i}\right) \geq p .
$$

Since $\mu^{i}\left(C \mid \Pi_{k}^{i}\right) \geq p$ for every $i \in I$, we deduce that

$$
\mu^{i}\left(C \mid \Pi^{i}\left(\omega^{\prime}\right)\right)=\mu^{i}\left(C \mid \Pi_{k}^{i}\right) \geq p .
$$

\section{Proof of Theorem 3.}

(I) implies (II).

Since $\delta>0$, there is no common prior, hence there exists a bet $\bar{B}$, i.e., w.l.o.g., $\bar{B}$ satisfies the property that for each state $\omega, E_{1}\left(\bar{B} \mid \Pi_{1}(\omega)\right)>0>$ $E_{2}\left(\bar{B} \mid \Pi_{2}(\omega)\right)$, meaning there is common knowledge that in the interim stage player 1 will be willing to buy the bet and player 2 will be willing to take the opposite side and sell. That is not enough to fashion a trade, as can be seen from Theorem 2; one needs 'some irrationality'.

Firstly, one can add $q \in \mathbb{R}_{+}$to the bet $\bar{B}$ at each state $\omega$ to form a random variable $B$ (strictly speaking it will not be a bet, as it is not zero sum) such that for each state $\omega, E_{1}\left(\bar{B} \mid \Pi_{1}(\omega)\right)>q>E_{2}\left(\bar{B} \mid \Pi_{2}(\omega)\right)$. 
By the assumption of $p$-overlapping, we may identify $\pi^{1}=\bigcup_{k \in K^{1}} \Pi_{k}^{1}$ and $\pi^{2}=\bigcup_{k \in K^{2}} \Pi_{k}^{2}$ that satisfy (1) and (2) of Definition 10. From this, there is common $p$-belief in $\pi^{1} \cap \pi^{2}$.

For each $i=1,2$ choose a subset of $\Omega$ denoted as $F^{j}$ such that $F^{j} \subseteq$ $\pi^{i} \backslash \pi^{j}, F^{j} \subsetneq \Pi^{i}(\omega)$ for any $\Pi^{i}(\omega)$, and $\mu^{i}\left(F^{j}\right) \leq \rho^{i}$ (and $\mu^{j}\left(F^{j}\right) \geq 0$ ).

We construct two pairs of strategies. First consider $\bar{s}^{1}$ and $\bar{s}^{2}$ as follows:

$$
\begin{aligned}
& \bar{s}^{1}(\omega, B, q)= \begin{cases}\text { 'buy' } & \text { if } \Pi^{1}(\omega) \cap\left(\pi^{1} \cup F^{1}\right) \neq \emptyset \\
\text { 'refrain' } & \text { otherwise; }\end{cases} \\
& \bar{s}^{2}(\omega, B, q)= \begin{cases}\text { 'sell' } & \text { if } \Pi^{2}(\omega) \cap\left(\pi^{2} \cup F^{2}\right) \neq \emptyset \\
\text { 'refrain' } & \text { otherwise, }\end{cases}
\end{aligned}
$$

The second pair of strategies is given by

$$
s^{i}(\omega, B, q)= \begin{cases}\text { 'buy/sell' } & \text { if } \Pi^{i}(\omega) \cap \pi^{i} \neq \emptyset \\ \text { 'refrain' } & \text { otherwise; }\end{cases}
$$

Note that in the set $\Pi^{i}(\omega) \cap \pi^{i}$, the strategies $\bar{s}^{i}(\cdot)$ and $s^{i}(\cdot)$ coincide. In $F^{i}$, the strategy $\bar{s}^{i}(\cdot)$ is suboptimal, i.e., $\bar{s}^{i} \notin R^{i}\left(B, q, \omega, s_{j}\right)$ for each $\omega \in F^{i}$, hence this is an irrational strategy for player $i$ at the states in $F^{i}$. In contrast, each of $s^{i}(\cdot)$ is a best reply to $\bar{s}^{j}$ at all $\omega \in \Omega$. We proceed now to show formally that $\left(s^{1}(\cdot), s^{2}(\cdot)\right)$ constitute a $\left(1-\rho^{1}, 1-\rho^{2}\right)$-rationality interim Nash Equilibrium.

Consider without loss of generality player 1:

- If $\Pi^{1}(\omega) \subseteq \pi^{1} \cap \pi^{2}$, then at any state in $\Pi^{1}(\omega)$ 'buy' is optimal for trader 1 , because his conditional expectation is for $B(\omega)$ to be greater than the offered price $q$, and trade takes place with probability 1 (since player 2 is willing to sell).

- For $\omega \notin \pi^{1} \cup \pi^{2}$, trade does not occur (as the players choose 'refrain').

- For $\omega \in \pi^{2} \backslash \pi^{1}$, trader 1 believes that trader 2 is willing to sell. Furthermore, trader 1 believes that trader 2 believes that trader 1's strategy calls on him (irrationally) to buy at states $\omega$ such that $\Pi^{1}(\omega) \cap$ $F^{1} \neq \emptyset$ (as player 2 believes that player 1's expectation at such an $\omega, E\left(B \mid \Pi^{1}(\omega) \cap\left\{\omega \mid s^{2}(\omega, B, q)=\right.\right.$ 'sell' $\left.\}\right)$, is less than $\left.q\right)$ and refrain otherwise.

- For $\omega \in \pi^{1} \backslash \pi^{2}$ 'buy' is still optimal for trader 1 , because by construction trader 1's expected value conditional on $\Pi^{1}(\omega)$ and on the event of trade (which happens on $\pi^{2}$ and $F^{2}$, even if trading is irrational on $F^{2}$ for player 2) is still greater than or equal to $q$.

Finally the result follows because $\Pi$ is connected. 
A symmetric argument shows that the rational strategy of player 2 is a best reply to "irrational" strategy of player 1 .

By construction, $\mu^{1}\left(F^{2}\right) \leq \rho^{1}$ and $\mu^{2}\left(F^{1}\right) \leq \rho^{2}$ (with $\rho^{i}>0$ for at least one $i$ ) hence $s(\omega, B, q)$ is a $\left(1-\rho^{1}, 1-\rho^{2}\right)$-rationality interim $\mathrm{NE}$ equilibrium.

That $Z(s, B, q)$ is common- $p$ belief at any $\omega \in \pi^{1} \cap \pi^{2}$ follows because it satisfies (A2) and $\pi^{1}$ and $\pi^{2}$ satisfy (A1).

Finally, Proposition 1 implies that $y^{1}-y^{2} \leq(1-p(1-\delta))\|B\|_{\infty}$.

(II) implies (I).

Suppose that there exists a proposed trade $B$, a price $q$, and strategies $s^{1}, s^{2}$ such that $Z(s, B, q)$ is heterogeneous common $p$-belief at $\omega \in \Omega$. By Proposition 2 there exist two sets $\pi^{1}$ and $\pi^{2}$ satisfying Condition (A1) and (A2) of that proposition. Condition (A1) implies Condition (1) of Definition 10.

Suppose next that Condition (2) fails to obtain. Then there exist nonempty index sets $K^{1^{\prime}} \subseteq K^{1}$ and $K^{2^{\prime}} \subseteq K^{2}$ such that both $\mu^{1}\left(\pi^{1^{\prime}} \Delta \pi^{2^{\prime}}\right)=0$ and $\mu^{2}\left(\pi^{1^{\prime}} \Delta \pi^{2^{\prime}}\right)=0$. But for any $\omega \in Z(s, B, q)$, 'buy' is a unique optimising action on $\Pi^{1}(\omega)$, and since trader 1 is rational in $Z(s, B, q)$, it follows that $s^{1}\left(\omega^{\prime}\right)=$ 'buy' for all $\omega^{\prime} \in \Pi^{1}(\omega)$. A similar statement holds for $s^{2}\left(\omega^{\prime}\right)=$ 'sell' for all $\omega^{\prime} \in \Pi^{2}(\omega)$. Hence, if both $\mu^{1}\left(\pi^{1^{\prime}} \Delta \pi^{2^{\prime}}\right)=0$ and $\mu^{2}\left(\pi^{1^{\prime}} \Delta \pi^{2^{\prime}}\right)=0$, if follows that for all $\omega \in \pi^{1^{\prime}} \Delta \pi^{2^{\prime}}, \Pi^{1}(\omega) \in \pi^{1^{\prime}}$ and 'buy' is trader 1's optimising action, and $\Pi^{2}(\omega) \in \pi^{2^{\prime}}$ and 'sell' is trader 2 's optimising action. Therefore at any $\omega \in \pi^{1^{\prime}} \Delta \pi^{2^{\prime}}$ there is common knowledge of strictly improving trade among rational traders, contradicting Theorem 2.

Proof of Theorem 4. The proof of Theorem 4 generally follows similar steps to those of the corresponding result of Neeman (1996b).

We start by slightly adapting the text of the lemma on page 94 of that paper, to incorporate non-common priors (the proof is similar to the original Lemma and omitted).

Lemma Let there be given two positive constants $c$ and $\psi$ and two random variables $e(\omega)$ and $B(\omega)$ such that $|e(\omega)|,|B(\omega)| \leq M / 2$ for all $\omega \in \Omega$. Let $T$ and $\Pi$ be two measurable subsets of $\Omega$ such that $\left.\mu_{i}(T \cup \Pi)>0\right)$ and $E_{\mu_{i}}(B \mid T \cup \Pi) \geq 0$. Then:

(1) There exists a $\delta>0$ such that for all $u \in U_{M, \phi}$ satisfying $\sup _{\{|x| \leq M\}}\left|u^{\prime \prime}(x)\right|<$ $\delta, E\left(u\left(e(\omega)+\mathbb{1}_{\omega \in T}(B(\omega)+c)\right) \mid \Pi\right)>E(u(e(\omega)) \mid \Pi) ;$ 
(2) Define $c(\hat{u}, \mu)$ such that $E\left(u\left(e(\omega)+\mathbb{1}_{\omega \in T}(B(\omega)+c(\hat{u}, \mu)) \mid \Pi\right)=\right.$ $E(u(e(\omega)) \mid \Pi)$. For all $\varepsilon>0$ there exists $a \delta>0$ such that for all $u \in U_{M, \psi}$ satisfying $\sup _{\{|x| \leq M\}}\left|u^{\prime \prime}(x)\right|<\delta, c(\hat{u}, \mu)<\varepsilon$.

Condition (1) says that provided that risk aversion of the players (as measured by $\left|u^{\prime \prime}().\right|$ is not too high, it is possible to increase the player's utility for any $\omega$ by a giving him a lottery compensated by some extra positive amount. Condition (2) says that such compensation is bounded.

(II) implies (I). Let sets $F^{1}$ and $F^{2}$ be generated as in the proof of Theorem 3 and let trade $B$ satisfy all the conditions specified in the statement and proof of Theorem 3. As before, since $\delta>0$ there exists a bet $\bar{B}$ such that $E_{1}\left(\bar{B}(\omega) \mid \Pi_{1}(\omega)\right)=y^{1}$ for all $\omega$ and $E_{2}\left(\bar{B}(\omega) \mid \Pi_{2}(\omega)\right)=y^{2}$ for all $\omega$, with $y^{1}>y^{2}$ w.l.o.g.

By the above Lemma, if traders are sufficiently risk tolerant, there exists a price in the interval $\left(y^{2}+C^{2}\left(\mu^{2}, u^{2}\right), y^{1}-C^{1}\left(\mu^{1}, u^{1}\right)\right)$, where $C^{i}\left(\mu^{i}, u^{i}\right)$ is given in (10), such that trade can weakly improve each player's utility. We now follow an argument similar to the proof of Theorem 3 to show that $s^{1}$ and $s^{2}$ constitutes a $(1-\rho)$-rationality Nash equilibrium, if the agents are sufficiently risk tolerant. Indeed, similarly to the proof of Theorem 3 , within $\pi^{1}$ agent 1 is rational and accepting $B$ at price $q$ is the optimal action (his expectation of $B$ is greater than $q$ and in addition $q$ is adjusted to cover a "risk premium"). On $F^{2}$ agent 1 believes that player 2's follows his irrational strategy and best replies with his optimal strategy to buy. On $\Omega \backslash\left(\pi^{1} \cup F^{1}\right)$ refraining from trade is the optimal action for trader 1 .

The same argument holds symmetrically for agent 2 , who is rational everywhere and believes that on $F^{1}$ he best replies to a suboptimal strategy of player 1 . Hence $s^{1}$ and $s^{2}$ constitute a $(1-\rho)$-rationality Nash equilibrium. In addition, $T(s, B, q)$ is heterogeneous common $p$-belief at any $\omega \in \pi^{1} \cap \pi^{2}$, by the same reasoning as in the proof of Theorem 3 .

(I) implies (II). The proof is similar to the proof of Theorem 3.

\section{REFERENCES}

Aumann, R. J. (1976), Agreeing to Disagree, Annals of Statistics, 4(6), 1236-1239.

Dow, J., V. Madrigal, and S. R. Werlang, (1990), Preferences, Common Knowledge and Speculative Trade, Fundacao Getulio Vargas Working Paper.

Grinblatt, M., and M. Keloharju (2009), Sensation Seeking, Overconfidence and Trading Activity, Journal of Finance 64, 549-578. 
Fagin, R., J. Y. Halpern, Y. Moses, and M. Y. Vardi (1995), Reasoning About Knowledge, MIT Press, Cambridge, MA.

Feinberg, Y. (2000), Characterizing Common Priors in the Form of Posteriors, Journal of Economic Theory 91, 127-179.

Gul, F. (1998), A Comment on Aumann's Bayesian View, Econometrica, 66, 923-927.

Heifetz, A. (2006), The Positive Foundation of the Common Prior Assumption, Games and Economic Behavior, 56 (1), 105-120.

Hellman, Z., and D. Samet (2012), How Common are Common Priors, Games and Economic Behavior, 74, 517-525

Milgrom, P. and N. L. Stokey, (1982), Information, trade and common knowledge, Journal of Economic Theory, (26), 17-27.

Monderer, D., and D. Samet (1992), Approximating Common Knowledge with Common Beliefs, Games and Economic Behavior, 1, 170-190.

Morris, S. (1995), Trade with Heterogenous Prior Beliefs and Asymmetric Information, Econometrica, 62, 1327-1347.

Neeman, Z. (1996a), Approximating Agreeing to Disagree Results with Common p-Beliefs, Games and Economic Behavior, 12, 162-164.

Neeman, Z. (1996b), Common Beliefs and the Existence of Speculative Trade, Games and Economic Behavior, 16, 77-96.

Rubinstein, A. (1975), Security Market Efficiency in an ArrowDebreu Economy, American Economic Review, 65, 812-824.

Samet, D. (1998), Common Priors and Separation of Convex Sets, Games and Economic Behavior, 24, 172-174.

Samet, D. (2000), Quantified Beliefs and Believed Quantities, Journal of Economic Theory, 95, 169-185.

Sebenius, J., and J. Geanakoplos (1983), Don't Bet on it: Contingent Agreements with Asymmetric Information, Journal of the American Statistics Association, 78, 424-426.

Tirole, J. (1982), On the Possibility of Speculation under Rational Expectations, Econometrica, 50 (5), 1163-1182.

Department of Economics, University of St. Gallen, SWitzerland; DePARTMENT OF ECONOMICS, BAR ILAN UNIVERSITY, ISRAEL 